\title{
Desarrollo de la competencia científica mediante el aprendizaje basado en proyectos y TIC en Educación Primaria
}

\author{
Eider Bilbao-Aiastui \\ eiderbilbao@opendeusto.es \\ Universidad de Deusto, España
}

\begin{abstract}
Resumen
En la presente investigación se analiza la integración del método de aprendizaje basado en proyectos, a través de las TIC como método emergente con impacto significativo en el aprendizaje. Se implementan dos unidades didácticas de la higiene de las manos a través de un método tradicional y un método de aprendizaje basado en proyectos y TIC como recurso educativo. Mediante un diseño cuasi experimental se analizan los datos de 39 alumnos de $2^{\circ}$ de primaria, aplicando el test $U$ de Mann Whitney, un cuestionario y la observación participante. Se resalta que el trabajo e integración de la metodología de aprendizaje basado en proyectos, a través de las TIC no mejora significativamente en el rendimiento académico. Sin embargo, se subrayan mejoras estadísticamente significativas en el reconocimiento de los contenidos, la participación activa, la colaboración, satisfacción y la motivación en los procesos de aprendizaje. A través de este método de aprendizaje y la utilización de las TIC, los estudiantes investigan de primera mano y reflexionan sobre el tema, realizando un aprendizaje significativo.
\end{abstract}

\section{Palabras clave}

Educación primaria; innovación educativa; aprendizaje basado en proyectos; tecnologías de la información y comunicación 


\title{
Development of scientific competence through the project based learning and ICT in Primary Education
}

\author{
Eider Bilbao-Aiastui \\ eiderbilbao@opendeusto.es \\ Universidad de Deusto, Spain
}

\begin{abstract}
This research analyses the integration of the project based learning method, through ICT as an emerging method with significant impact on learning. Two didactic units of hand hygiene are implemented through a traditional method and a project-based learning method and ICT as an educational resource. By means of a quasi experimental design, data from 39 students of 2nd grade of primary school are analyzed, applying the Mann Whitney U-test, a questionnaire and participant observation. It is highlighted that the work and integration of the project based learning methodology, through ICT, does not significantly improves academic performance. However, statistically significant improvements are highlighted in the content recognition, active participation, collaboration, satisfaction and motivation in learning processes. Through this learning method and the use of ICT, students investigate first-hand and reflect on the subject by doing significant learning.
\end{abstract}

\section{Keywords}

Primary education; educational innovation; project based learning; information and communication technologies 


\section{Introducción}

Las nuevas Tecnologías de la Información y la Comunicación (TIC) han provocado una revolución en todos los ámbitos y sectores de la sociedad, que ahora se considera la Sociedad de la Información y el Conocimiento. Barroso et al. (2007) destacan que la sociedad de la información y la comunicación pone fin a la era industrial y da comienzo a la era digital. Además, la sociedad actual se dirige hacía una Cuarta Revolución Industrial donde los ciudadanos necesitarán competencias digitales (Williamson, Potter \& Eynon, 2019; Brugia \& Zukersteinova, 2019). Domínguez (2016) subraya que la red ya no es solo un lugar donde buscar información, es decir, también es un espacio donde se puede expresar, interactuar y compartir información. Johnson, Adams Becker, Estrada \& Freeman (2015) explican la importancia de las aplicaciones tecnológicas y las tecnologías futuras y emergentes (FET), ya que constituyen el trasfondo de los nuevos desafíos sociales y científicos. Además, Area (2015) indica que la escuela del siglo XXI debe transformar los materiales de enseñanza, así como las metodologías de aprendizaje, a fin de responder a las nuevas necesidades de enseñanza y aprendizaje. Como consecuencia de la importancia de las competencias digitales, distintos organismos e instituciones dan un nuevo enfoque al sistema educativo, en el cual enumeran las competencias básicas y en todas se incluye la competencia digital (European Commision, 2016; INTEF, 2017; Ley Orgánica, 2006; Ley Orgánica, 2013; OCDE, 2005; UNESCO, 2011). Asimismo, la European Commision (2006) señala ocho competencias básicas que debe tener cualquier ciudadano para el aprendizaje permanente, siendo una de ellas la competencia digital.

Según Salinas (2008) las TIC son una revolución para el concepto de educación tradicional, ya que permiten implementar acciones innovadoras en cualquier ámbito y nivel educativo. Del mismo modo, Yang \& Kwok (2017) destacan el uso de las TIC para facilitar la enseñanza y el aprendizaje tanto de los profesores como de los alumnos. Salinas (2008) apoya que los maestros tengan un cambio significativo, ya que se convierten en facilitadores y guías del proceso de aprendizaje. Sin embargo, no sólo el maestro experimenta un cambio de rol, los estudiantes asumen un papel activo en su proceso de enseñanza, aumentando su autonomía (Salinas, 2008). Según Barroso et al. (2007) el uso de las TIC en la educación proporciona las siguientes ventajas:

- Amplia gama de información

- Creación de entornos flexibles para el aprendizaje

- Eliminación de las barreras espacio temporales entre el profesor y los estudiantes

- Aumento de la competencia comunicativa

- Mejora de los escenarios y entornos interactivos

- Aprendizaje independiente, autoaprendizaje y aprendizaje colaborativo

- Nuevas posibilidades para la orientación y tutoría de los estudiantes

- Aprendizaje a lo largo de toda la vida

- Rompe con los escenarios clásicos de formación, limitados a las instituciones escolares

Pérez y Tejedor (2016) indican que un proyecto es el eje de la educación escolar, que tiene un plan de acción y permite alcanzar un determinado resultado, combinando el estudio empírico y el investigativo. Sin embargo, el método por proyectos es una estrategia de aprendizaje basada en proyectos $(A B P)$ en la que los alumnos diseñan, aplican y evalúan proyectos que tienen una aplicación en el mundo real más allá del aula escolar (Domínguez \& Jaime, 2010; Helle, Tynjälä \& Olkinuora, 2006; Thomas, 2000). El mismo es una estrategia metodológica globalizadora, ya que implica un diálogo entre tres elementos para lograr un aprendizaje significativo: los intereses de los estudiantes, el currículo establecido y las necesidades y la realidad del contexto en el que se 
desarrolla la acción educativa (Blanchard, Glower, Jiménez y Muzás, 2014). Helle et al. (2006) y Powell \& Weenk (2003) destacan que el ABP es un método de enseñanza centrado en el alumno que está diseñado para desarrollar el trabajo en grupo del alumno, las aptitudes interdisciplinarias, el pensamiento crítico, la comunicación interpersonal y las competencias de gestión de proyectos. Asimismo, el ABP fomenta que los estudiantes se involucren en el trabajo colaborativo en lugar de escuchar y leer las nociones (Fernandes, 2014; Michaelsen, Knight \& Fink 2004). Del mismo modo, los estudiantes tienen más oportunidades de interactuar con sus compañeros y se enriquecen más con el trabajo de los proyectos. Por lo tanto, los estudiantes utilizan las TIC en la aplicación del ABP (Heo, Lim \& Kim, 2010).

Además, este tipo de aprendizaje, según Blanchard et al. (2014), Pérez y Tejedor (2016) y Shih \& Tsai (2017), tiene varios beneficios:

- Aumenta la motivación y la autoestima

- Desarrolla el aprendizaje experimental, investigativo y reflexivo

- Aumenta las habilidades sociales y de comunicación

- Proporciona oportunidades de colaboración para construir conocimiento

- Ofrece conexiones en diferentes disciplinas entre la escuela y el mundo del trabajo

- Prepara a los estudiantes para el trabajo

Sin embargo, para aprovechar los beneficios del ABP, se deben respetar sus fases. Pérez y Tejedor (2016) indican que dicha metodología consta de ocho fases: definición de competencias, contextualización y diagnóstico, enmarcado, creación de equipos de trabajo, construcción de un problema específico, enfoque estratégico, ejecución y evaluación.

Según Heo et al. (2010) y Sendag \& Odabasi (2009), la enseñanza por proyectos no requiere específicamente el uso de tecnologías; sin embargo, al utilizar las TIC en este método de enseñanza, puede reducir los costos de aprendizaje, mejorar la eficacia del aprendizaje y crear más oportunidades (Heo et al., 2010; Sendag \& Odabasi, 2009). Del mismo modo, los estudiantes tienen más oportunidades de interactuar con sus compañeros y se enriquecen más con el trabajo de los proyectos.

En la siguiente sección se describen los aspectos metodológicos de la investigación realizada y en la tercera sección se muestra el análisis de los resultados. El documento termina con la sección de discusión y las conclusiones obtenidas.

\section{Importancia de las preguntas de estudio e investigación}

Si bien algunas investigaciones han propuesto que el aprendizaje de los estudiantes es mejor en un entorno de aprendizaje de método por proyectos utilizando las TIC en comparación con las aulas tradicionales, la cuestión sigue abierta a debate. En este sentido, el examen del efecto del ABP utilizando las TIC en el rendimiento de los estudiantes de la escuela primaria, puede contribuir tanto a la alfabetización como a los estudios relacionados con la formación de los estudiantes de la escuela primaria, ya que no está clara la contribución del ABP utilizando las TIC en el rendimiento de los estudiantes de la escuela primaria. Por lo tanto, el estudio proporciona resultados significativos para el aspecto psicosocial de las aulas que promueven o inhiben el aprendizaje en la formación de los estudiantes de primaria. 
El objetivo general es conocer si existen relaciones directas entre los resultados pedagógicos alcanzados tras la incorporación y utilización del ABP y las TIC en la enseñanza de las Ciencias Naturales en el aula de Educación Primaria.

De este objetivo general surgen otros dos objetivos específicos:

- Analizar si existen relaciones directas entre los resultados pedagógicos alcanzados tras la incorporación y utilización del APB y las TIC

- Conocer la satisfacción y motivación de los alumnos sobre el proceso de enseñanzaaprendizaje

\section{Metodología}

\section{a. Diseño de la investigación}

En el estudio se utiliza un diseño de investigación cuasi experimental en el que se aplicó la instrucción tradicional en el grupo de control y el grupo experimental aplica la instrucción diseñada según el ABP utilizando TIC. También, se lleva a cabo en los dos grupos un análisis descriptivo de un cuestionario y observación participante.

\section{b. Contexto educativo}

El estudio se lleva a cabo en una escuela concertada en Bizkaia, que se encuentra en el País Vasco, España. La escuela ofrece 3 niveles de educación: educación infantil, educación primaria y educación secundaria obligatoria. La misma tiene 40 profesores y 3 empleados administrativos. El mismo, forma a 700 estudiantes y participa en el proceso de Bolonia, ya que tiene como objetivo lograr mejores niveles en todas las áreas de la educación.

La enseñanza elemental, en la que se estudia, consta de 6 niveles y cada nivel tiene dos clases (AB). En la escuela se forma a los estudiantes para que obtengan los conocimientos científicos y las habilidades necesarias para su vida. Por lo tanto, todos los cursos se llevan a cabo de acuerdo con el programa marco establecido por el Gobierno español. La duración de la formación académica anual se determina en 3 secciones de 3 meses.

\section{c. Información del curso}

El estudio se lleva a cabo en dos aulas de $2^{\circ}$ año de educación primaria dentro de la asignatura de Ciencias Naturales que se imparte durante tres horas a la semana. En el aula tradicional, el aprendizaje se realiza a través de libros de texto y de instrucción directa, mientras que en el aula experimental se utiliza el método de ABP a través de las TIC. Después de la enseñanza tradicional, se espera que los estudiantes practiquen lo que han aprendido en su vida cotidiana sin haber hecho ninguna práctica durante la formación. Por lo tanto, con el nuevo enfoque experimental se crea una nueva oportunidad para que los estudiantes pongan en práctica los contenidos aprendidos durante la formación y en su vida cotidiana.

Además, los objetivos del curso son asegurar que los estudiantes apliquen diferentes estrategias y métodos de aprendizaje, comprendan y apliquen los principios de la instrucción planificada, utilicen materiales e instrumentos de aprendizaje apropiados y tomen conciencia de los deberes y responsabilidades. 


\section{d. Participantes}

En la investigación participan dos clases de enseñanza de Educación Primaria, se elige una clase como grupo experimental y otra clase como grupo de control aleatorio. No se informa a los estudiantes sobre el método de instrucción a utilizar antes de aplicar el curso. La muestra consta de 39 estudiantes en total, 20 chicas $(51,28 \%)$ y 19 chicos $(48,72 \%)$, todos entre los 7 y 8 . Por un lado, el grupo de control cuenta con 19 estudiantes, de los cuales 10 son niñas y 9 niños. Por otro lado, el grupo experimental consta de 20 estudiantes, de los cuales 10 son niñas y 10 niños. Cabe destacar que no hay diferencias significativas entre los dos grupos. Todos los alumnos poseen y utilizan computadoras durante las lecciones en la escuela y tienen acceso a diversas herramientas TIC (Google Apps, Hotmail...) para apoyar su aprendizaje durante las lecciones.

La selección aleatoria de los estudiantes no es posible, ya que los maestros eligen dos grupos en el mismo curso de acuerdo con la igualdad de los grupos, teniendo en cuenta el rendimiento de los estudiantes en escritura, lectura y comprensión. Por ello, la muestra del estudio es no probabilística e intencional.

\section{e. Instrumentos}

En los diseños cuasi experimentales los grupos no se forman aleatoriamente, así se garantiza un control sobre la mayor parte de las fuentes de invalidez. También son más accesibles en contextos educativos por la dificultad de obtener muestras aleatorias.

El estudio utiliza, por un lado, un cuestionario que analiza tres dimensiones: el rendimiento académico, la satisfacción y la motivación de los estudiantes. Para el rendimiento académico se utilizan 7 preguntas cerradas con alternativas de respuestas no ordenadas ( $\mathrm{Si}$, No, No lo sé). Son preguntas de escala nominal, donde se espera que el participante elija una de las alternativas ofrecidas como respuesta. Se utiliza este tipo de pregunta debido a su capacidad de generar datos cuantitativos y de incrementar la precisión con la que los participantes informan. Además, reducen los errores de compresión y de singularidad, controlando la dispersión. En cuanto a las dimensiones de satisfacción y motivación se utiliza 1 pregunta cerrada con alternativa de respuesta ordenada en cada dimensión, utilizando la escala de Likert ( 1 al 10) siendo 1 totalmente en desacuerdo y 10 totalmente de acuerdo adaptada para niños. Son dos preguntas basadas en una escala fija, donde cada dimensión queda ordenada en una secuencia de puntos arbitrarios de menor a mayor intensidad. Esta escala facilita el uso, ya que, los resultados pueden ser transformados fácilmente en porcentajes. Mediante la herramienta se aportan datos descriptivos, a partir de una escala y mediante un enfoque cuantitativo. Creswell (2003), destaca que este tipo de investigación pretende describir la experiencia individual en entornos particulares. Asimismo, la fiabilidad calculada a través del coeficiente de alfa de Cronbach en el cuestionario ofrece un 0,909 por lo que se considera aceptable (Hair, Anderson, Tatham \& Black, 1998).

Por otro lado, se desarrolla un enfoque cualitativo con la técnica de observación sistemática. La observación sistemática es una metodología de investigación donde se puede acceder directamente a lo que los alumnos han experimentado mientras realizan las actividades. Por ello, este método de recopilación de datos en la intervención educativa, asegura que los datos se recogen con la interacción directa del investigador con los participantes y evidencia y apoya la validez de los datos.

\section{f. Implementación del proyecto}

En España, el marco curricular se basa en contenidos, criterios de evaluación y estándares de aprendizaje en la escuela primaria (Ministerio de Educación y Cultura y Deporte MECD, 2014) y las 
competencias clave a lo largo de la vida (European Parliament and council, 2006). Para realizar el proyecto se ha tenido en cuenta el marco normativo y los elementos curriculares.

La intervención se lleva a cabo en dos aulas de $2^{\circ}$ de educación primaria, durante el curso académico 2017-2018. En cada aula se realizan 6 sesiones de 50 minutos en el área curricular de Ciencias Sociales. Las dos intervenciones son realizadas en lengua vasca. Es recomendable plantear una metodología didáctica centrada en el alumno (Ausubel, Novak y Hanesian, 1983) basada en el aprendizaje colaborativo (Johnson, 2003; Johnson, 2006) asumiendo roles y fomentando un trabajo activo y dinámico en el aula.

En el grupo de control, el aprendizaje se realiza a través de libros de texto y de instrucción directa, a través de un método tradicional. En este grupo el alumno escucha la materia y realiza las fichas oportunas en las 6 sesiones. Los contenidos que se dan en el grupo de control son: localización de las bacterias, necesidad de la higiene de las manos, medidas de prevención de la higiene de las manos y reflexión sobre la higiene de las manos.

Por otro lado, en el grupo experimental, el aprendizaje se realiza mediante el ABP y las TIC. Las posibilidades de compartir los contenidos a través de las mismas introducen la capacidad de trabajar con elementos multimedia, juegos y así facilitan el trabajo de determinados contenidos. También, se señalan ventajas mediante estos recursos en lo que se refiere al desarrollo de las habilidades relativas a la competencia digital y a la comprensión de los contenidos. Asimismo, se fomentan habilidades de pensamiento crítico, pensamiento comprensivo y análisis de contenidos. Por último, las posibilidades interactivas son más enriquecedoras, ya que el alumnado, manipula objetos y trabaja con los iguales en todo momento.

En la siguiente tabla 1 se presenta las sesiones y contenidos del grupo experimental:

\begin{tabular}{|c|c|}
\hline Sesión (50 min) & Contenidos \\
\hline 10 sesión & $\begin{array}{ll}\text { - } & \text { Presentación del proyecto } \\
\text { - } & \text { Localización de las bacterias y como son las mismas } \\
\text { - } & \text { Necesidad de higiene de las manos }\end{array}$ \\
\hline $2^{\circ}$ sesión & $\begin{array}{ll}\text { - } & \text { Experimento en grupos } \\
\text { - } & \text { Reflexión sobre el experimento y la vida real }\end{array}$ \\
\hline $3^{\circ}$ sesión & $\begin{array}{l}\text { - } \quad \text { Canción de la higiene de las manos } \\
\text { - } \quad \text { Medidas de prevención de la higiene de las manos } \\
\text { - } \quad \text { Cuestionario al experto }\end{array}$ \\
\hline $4^{\circ}$ sesión & $\begin{array}{ll}\text { - } & \text { Presentación del cuestionario realizado al experto } \\
\text { - } & \text { Reflexión en grupo sobre las respuestas } \\
\text { - } & \text { Debate sobre la higiene de las manos }\end{array}$ \\
\hline $5^{a}$ sesión & $\begin{array}{ll}\text { - } & \text { PowerPoint en grupos } \\
\text { - } & \text { Reflexión sobre la higiene de las manos } \\
\text { - } & \text { Debate sobre la higiene de las manos }\end{array}$ \\
\hline $6^{\circ}$ sesión & - Cuestionario \\
\hline
\end{tabular}

Tabla 1. Sesiones y contenidos del grupo experimental.

Fuente: elaboración propia.

En el siguiente enlace se puede observar la implementación de la unidad didáctica del grupo experimental: https://eiderpeder.wixsite.com/eskuak2 


\section{Análisis de los resultados}

Atendiendo a las tres dimensiones y la observación sistemática mencionadas en el marco metodológico, se presentan los resultados obtenidos en cada una de ellas, teniendo en cuenta el grupo de control y el grupo experimental. Para la recogida, el análisis y la presentación de los datos se ha utilizado las herramientas SPSS (Statistical Package for Social Sciences) y Excel.

\section{a. Dimensión del rendimiento académico 1}

En lo que respecta a inferencia estadística en la dimensión del rendimiento académico, se aplica una prueba no paramétrica en la que se analizan muestras independientes y se valoran los datos obtenidos, a partir de una comparación de medias en cuanto a ítems correctos del grupo de control y el grupo experimental (tabla 2). Hay que subrayar, que esta dimensión tiene 7 ítems, es decir, la puntuación máxima es de 7 ítems correctos equivalente a un 10. Por ello, se comprueba que el grupo experimental obtiene una media superior $(5,650)$ con un porcentaje de aciertos del $(\% 81)$ y que el grupo control obtiene una media de $(4,526)$ con un porcentaje de aciertos del $(\% 65)$. También, se observa en esta tabla la desviación típica de cada grupo.

\begin{tabular}{llcccc}
\hline & \multicolumn{3}{c}{ Estadísticas de grupo } & \\
\hline & Grupo & $N$ & Media & $\begin{array}{c}\text { Desv. } \\
\text { Desviación }\end{array}$ & $\begin{array}{c}\text { Desv. Error } \\
\text { promedio }\end{array}$ \\
\hline $\begin{array}{l}\text { Rendimiento } \\
\text { académico }\end{array}$ & Grupo Control & 19 & 4,5263 & 1,74383 &, 40006 \\
\cline { 2 - 5 } & Grupo Experimental & 20 & 5,6500 & 1,08942 &, 24360 \\
\hline
\end{tabular}

Tabla 2. Medias del rendimiento académico del grupo de control y el grupo experimental.

Fuente: elaboración propia.

Por otro lado, mediante esta herramienta se obtiene la d de Cohen en cuanto a la dimensión del rendimiento académico, el cual es de $(0,777)$ (tabla 3 ). Por ello, se estima que el tamaño de efecto de las muestras independientes en esta dimensión es grande (Cohen,1998).

\section{Tamaños de efecto de muestras independientes}

Intervalo de confianza al $95 \%$

\begin{tabular}{llcccc} 
& & Standardizera & $\begin{array}{c}\text { Estimación de } \\
\text { puntos }\end{array}$ & Inferior & Superior \\
\hline $\begin{array}{l}\text { Rendimiento } \\
\text { académico }\end{array}$ & d de Cohen & 1,44528 &,- 777 & $-1,425$ &,- 120 \\
\cline { 2 - 5 } & Corrección de Hedges & 1,47542 &,- 762 & $-1,396$ &,- 118 \\
\cline { 2 - 5 } & Delta de Glass & 1,08942 & $-1,031$ & $-1,728$ &,- 313 \\
\hline
\end{tabular}

Tabla 3. Tamaños de efecto de muestras independientes.

Fuente: elaboración propia.

Asimismo, en lo que respecta a inferencia estadística en esta dimensión, se aplica una prueba no paramétrica analizando muestras independientes y valorando las diferencias del grupo de control y el grupo experimental mediante el test $U$ de Mann de Whitney que tiene un valor de 117,000, un valor $Z$ de -2,102 y rangos que se detallan en la tabla 4. En definitiva, la significación del 0,036 es 
superior a 0,01 que es el nivel de significación de referencia en esta investigación. A partir de estos datos se interpreta que no hay diferencias significativas respecto al rendimiento académico, por lo que se acepta la hipótesis nula: no se detecta que la aplicación de la metodología APB y las TIC mejore el rendimiento académico.

\begin{tabular}{|l|l|l|l|}
\hline GC_GE & N & Rango promedio & Suma de rangos \\
\hline Control & 19 & 16,16 & 307,00 \\
\hline Experimental & 20 & 23,65 & 473,00 \\
\hline Total & 39 & & \\
\hline
\end{tabular}

Tabla 4. Rendimiento académico, Rangos. Test U de Mann de Whitney.

Fuente: elaboración propia.

A continuación, se presentan la cantidad de ítems correctos que ha realizado cada grupo (figura 1).

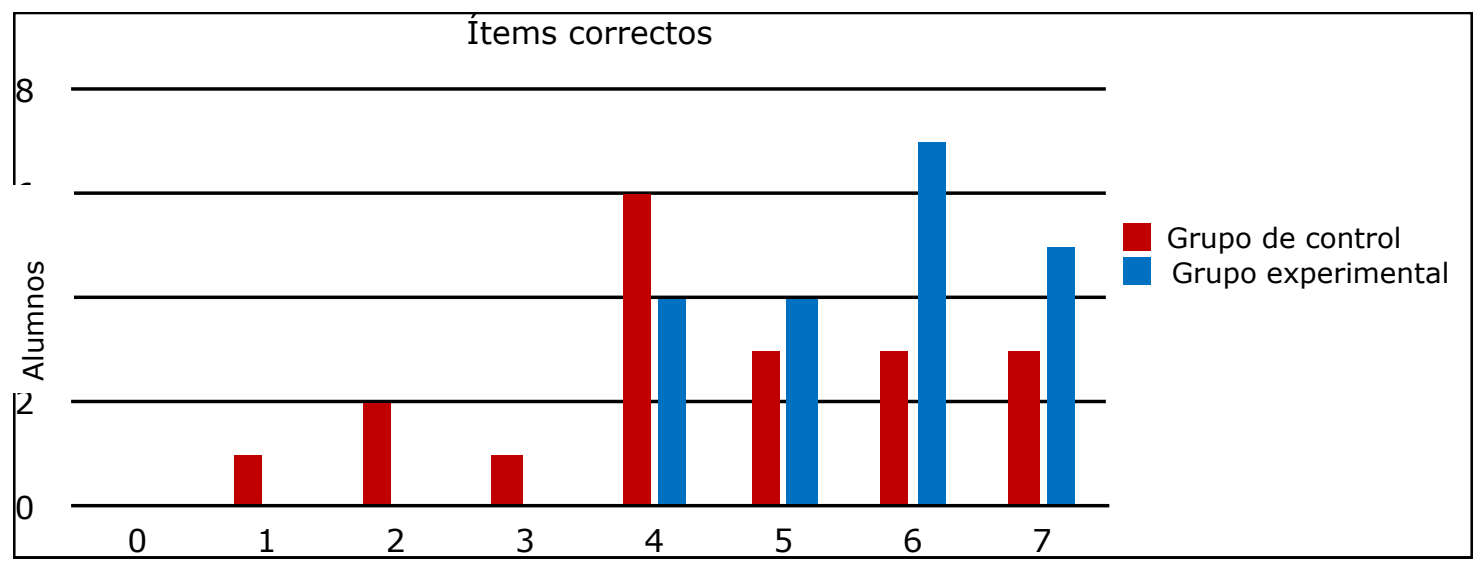

Figura. 1. Ítems correctos que realiza cada grupo teniendo en cuenta el alumnado.

Fuente: elaboración propia.

La figura 1 nos presenta que en el grupo experimental todos los alumnos obtienen de 4 a 7 ítems correctos, es decir todos aprueban el cuestionario final. A diferencia de estos, en el grupo de control un alumno obtiene 1 ítem correcto, dos alumnos de 2 y uno de 3, es decir 6 alumnos suspenden el cuestionario. La mayoría de los alumnos del grupo de control obtienen 4 ítems correctos, sin embargo, en el grupo experimental la mayoría obtiene 6 ítems correctos, es decir un sobresaliente.

Asimismo, se destaca que en el grupo experimental el $100 \%$ de los alumnos aprueban el cuestionario final a diferencia del grupo de control.

Por ende, se subraya que aunque no se destaque una mejora estadísticamente significativa, si se obtienen mejoras significativas en el rendimiento académico mediante el ABP y las TIC.

\section{b. Dimensión de la satisfacción 2}

Del mismo modo que en la dimensión anterior, a partir de una comparación de medias se obtiene las medias de satisfacción del grupo de control y el grupo experimental (tabla 5). Esta dimensión consta de 1 ítem de escala Likert ( 1 totalmente en desacuerdo y 10 totalmente de acuerdo). Mediante la misma, el grupo experimental obtiene una media superior $(7,150)$ con un porcentaje de satisfacción del $(\% 72)$ y que el grupo control obtiene una media de $(4,578)$ con un porcentaje de satisfacción del (\%46). También se observa en esta tabla la desviación típica de cada grupo. 


\begin{tabular}{|c|c|c|c|c|c|}
\hline \multicolumn{6}{|c|}{ Estadísticas de grupo } \\
\hline & Grupo & $\mathrm{N}$ & Media & $\begin{array}{c}\text { Desv. } \\
\text { Desviación }\end{array}$ & $\begin{array}{c}\text { Desv. Error } \\
\text { promedio }\end{array}$ \\
\hline \multirow[t]{2}{*}{ Satisfacción } & Grupo Control & 19 & 4,5289 & 1,83533 & 42105 \\
\hline & Grupo Experimental & 20 & 7,1500 & 1,42441 & 31851 \\
\hline
\end{tabular}

Tabla 5. Medias de la satisfacción del grupo de control y el grupo experimental.

Fuente: elaboración propia.

En la siguiente figura 2 se indican los datos sobre el nivel de satisfacción de los alumnos teniendo en cuenta la metodología utilizada (figura 2).

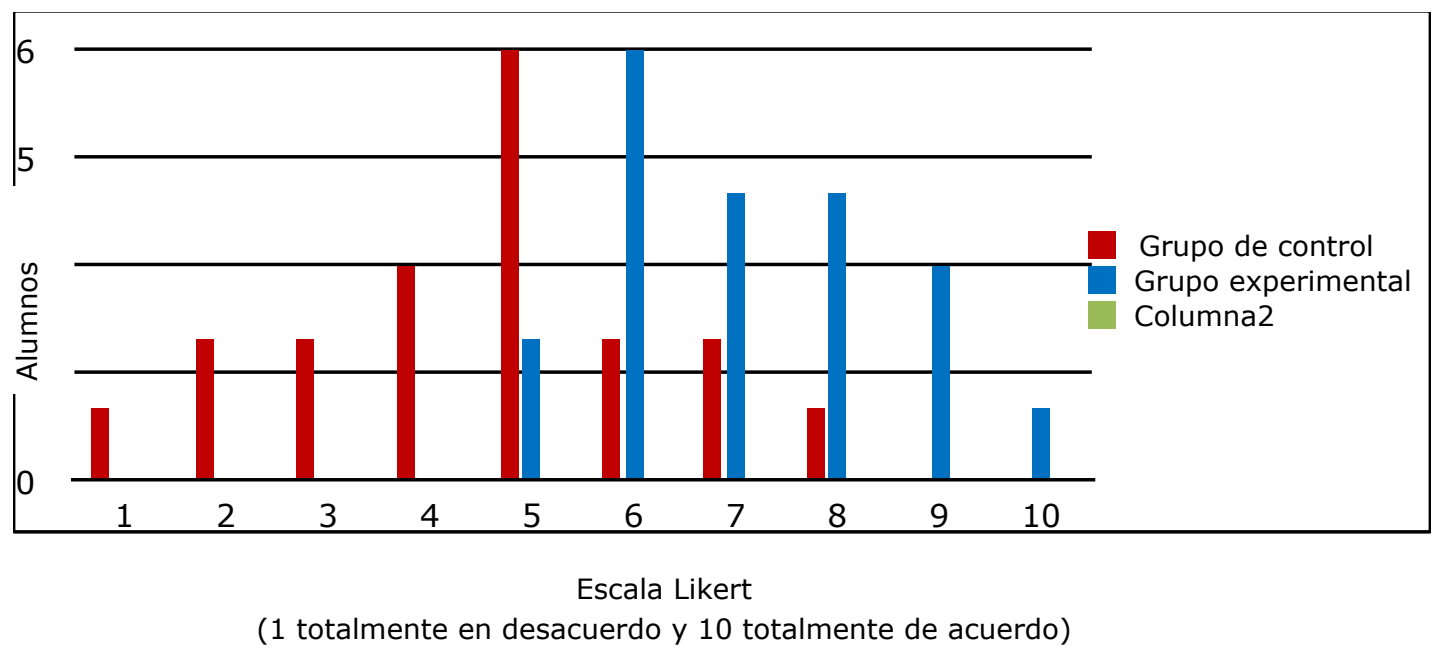

Figura. 2. Nivel de satisfacción de cada grupo teniendo en cuenta el alumnado.

Fuente: elaboración propia.

La figura 2 nos indica que ningún alumno del grupo de control está totalmente de acuerdo con la satisfacción de la metodología. Por ende, la mayoría de estos alumnos indican un 5 en la escala Likert teniendo en cuenta la satisfacción de la metodología siendo la misma la moda de estos alumnos. Por otro lado, en el grupo experimental ningún alumno indica que está totalmente en desacuerdo con la satisfacción de la metodología empleada. Es más, la mayoría de los alumnos indican un 6 en la escala Likert, siendo la misma la moda de estos alumnos y muchos de ellos afirman estar muy satisfechos con la metodología empleada. Asimismo, se destaca que en el grupo experimental el $100 \%$ de los alumnos aportan valores positivos respecto a la satisfacción del uso del ABP a través de las TIC.

En general, los valores que se presentan en esta dimensión por el grupo experimental son bastantes positivos a diferencia del grupo de control. Por tanto, desde un enfoque descriptivo, se valora muy positivamente la integración de la intervención descrita por parte de los sujetos, propiciando abordar contenidos con un enfoque activo, útil y satisfactorio.

Asimismo, mediante el test $\mathrm{U}$ de Mann de Whitney se obtiene la significación del 0,00 siendo menor a 0,01 y de este modo se detalla una mejora estadísticamente significativa en el mismo, por lo que los alumnos mejoran claramente en satisfacción al llevar a cabo las mencionadas prácticas. 


\section{c. Dimensión de la motivación 3}

Del mismo modo que en las anteriores dimensiones, a partir de una comparación de medias se obtiene las medias de motivación del grupo de control y el grupo experimental (tabla 6). Esta dimensión consta de 1 ítem de escala Likert ( 1 totalmente en desacuerdo y 10 totalmente de acuerdo). Mediante la misma, el grupo experimental obtiene una media superior de $(7,550)$ con un porcentaje de satisfacción del $(\% 76)$ y el grupo control obtiene una media de $(4,578)$ con un porcentaje de satisfacción del (\%48). También se observa en esta tabla la desviación típica de cada grupo.

\begin{tabular}{llcccc}
\hline & \multicolumn{5}{c}{ Estadísticas de grupo } \\
\hline & Grupo & N & Media & $\begin{array}{c}\text { Desv. } \\
\text { Desviación }\end{array}$ & $\begin{array}{c}\text { Desv. Error } \\
\text { promedio }\end{array}$ \\
\hline Motivación & Grupo Control & 19 & 4,7895 & 1,75052 &, 40160 \\
\cline { 2 - 6 } & Grupo Experimental & 20 & 7,5500 & 1,46808 &, 32827 \\
\hline
\end{tabular}

Tabla 6. Medias de la motivación del grupo de control y el grupo experimental.

Fuente: elaboración propia.

En la siguiente figura 3 se indican los datos sobre el nivel de motivación de los alumnos teniendo en cuenta la metodología utilizada (figura 3).

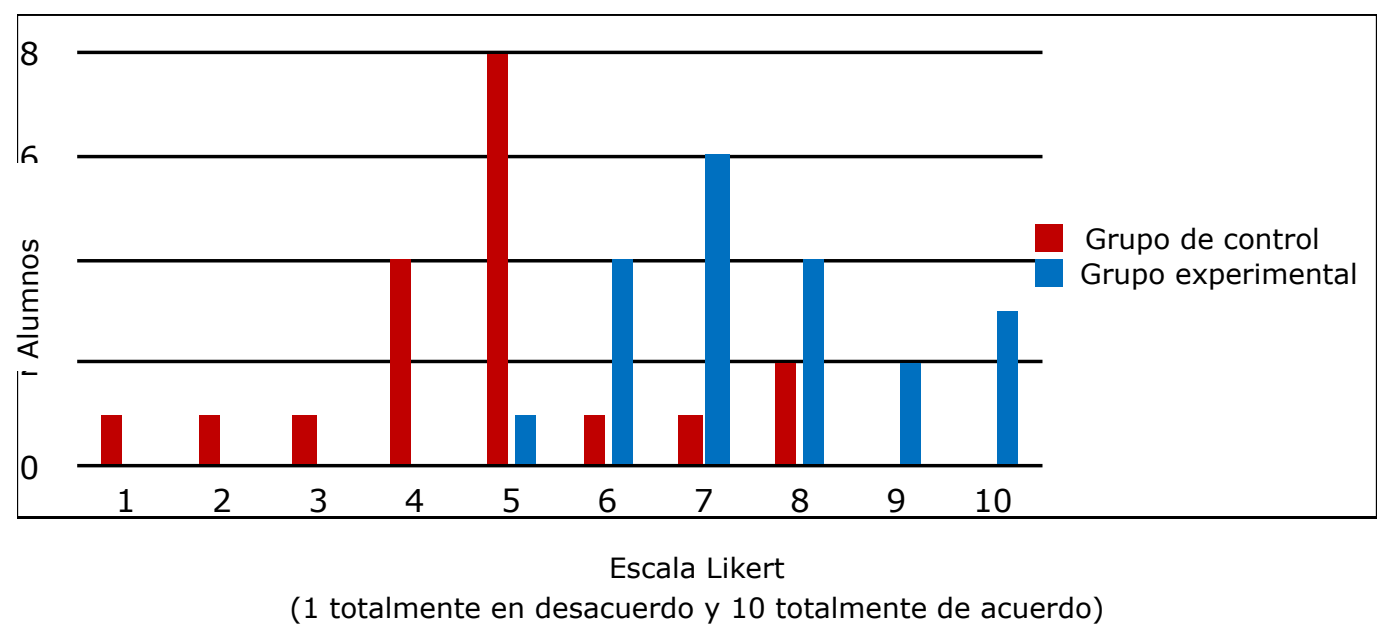

Figura. 3. Nivel de motivación de cada grupo teniendo en cuenta el alumnado.

Fuente: elaboración propia.

Como se refleja en la figura 3 ningún alumno del grupo de control esta totalmente de acuerdo y la mayoría marca esta pregunta con un 5 , teniendo en cuenta la motivación de la metodología siendo la misma la moda de este grupo. Por otro lado, en el grupo experimental ningún alumno dice estar en totalmente desacuerdo, la mayoría de los alumnos indican con un 7 la motivación de la metodología siendo la misma la moda y tres alumnos puntuan esta pregunta con un 10. Asimismo, se destaca que en el grupo experimental el $100 \%$ de los alumnos aportan valores positivos respecto a la motivación del uso del ABP a través de las TIC.

En general, los valores que se presentan en esta dimensión por el grupo experimental son bastantes positivos a diferencia del grupo de control. Por tanto, desde un enfoque descriptivo, se valora muy positivamente la integración de la intervención descrita por parte de los sujetos, propiciando abordar contenidos con un enfoque activo, divertido y motivador. 
Asimismo, mediante el test $\mathrm{U}$ de Mann de Whitney se obtiene la significación del 0,00 siendo menor a 0,01 y de este modo se detalla una mejora estadísticamente significativa en el mismo, por lo que los alumnos mejoran claramente en motivación al llevar a cabo las mencionadas prácticas.

\section{d. Observación sistemática}

La observación sistemática se concreta como una técnica estructurada e intencionada de mirar y examinar datos, objetos o fenómenos sin modificarlos (Sáez-López y Cózar, 2017). Este método es fundamental para el trabajo de campo, ya que favorece a la extracción de información valiosa en el contexto socio educativo.

El estudio opta por la observación participante, donde el investigador interviene activamente en las actividades de estudio, con ciertas características, como subjetividad, empatía y comprensión. La observación participante recopila datos a lo largo de la intervención educativa con la interacción directa del investigador y los estudiantes. Como se trata de observación sistemática estructurada, se clasifican sujetos u objetos en clases o categorías predefinidas.

La fiabilidad de este proceso de clasificación se establece mediante preguntas a dos evaluadores y así certificando independientemente esta clasificación con el mismo conjunto de objetos. Por ende, se participa en una confiabilidad entre evaluadores comparando su grado de acuerdo. La prueba utilizada para esta medición es la kappa de Cohen, con el profesor (en cada clase) y el investigador como evaluadores, teniendo en cuenta el nivel de acuerdo. Este valor de correlación de kappa puede variar de -1 a 1 y teniendo en cuenta el mismo los evaluadores acuerdan solamente aceptar los ítems superiores a 0,60 en esta investigación, es decir, todos los ítems por debajo de este valor son eliminados. Cohen (1960) acepta valores desde 0,60 como aceptables y sustanciales.

Las unidades de observación de las listas de control tienen una gran coherencia con los indicadores del estudio: aprendizaje activo, contenidos de higiene de las manos, utilidad, diversión, trabajo en grupo, rendimiento, evaluación y resultados. Por ende, los valores del instrumento son coherentes con las dimensiones, indicadores e ítems del cuestionario administrado. Las valoraciones relativas a aprendizaje activo y contenidos aportan valores superiores a 8 , mientras que la utilidad obtiene un valor de 7. El valor más alto con un 9 se relaciona con la diversión.

Asimismo, mediante la observación sistemática, se validan los datos adquiridos, ya que, el grupo experimental esta más receptivo durante la unidad didáctica (motivado, activo, colaborativo...) y obtiene mejores resultados en cuanto a todas las dimensiones. Por ejemplo: realizando el experimento, todos los integrantes de los grupos quieren hacer los requisitos, se ayudan unos a otros y estan muy entusiasmados en todo el proceso. También, cantando la canción de la higiene de las manos, los alumnos optan por querer cantar más veces, ya que les parece muy bonita, entretenida y una manera muy simple de aprender cómo limpiarse las manos. Por esta razón, se subraya que el grupo experimental esta más motivado en toda la unidad didáctica y fomenta la satisfacción personal y el ABP a través de las TIC.

\section{Discusión y conclusiones}

Varias investigaciones analizan la integración del ABP con las TIC, destacando numerosos beneficios y ventajas, como la motivación, la reflexión, la investigación, las habilidades comunicativas, la colaboración y las conexiones con las diversas disciplinas (Blanchard et al., 2014; Perez y Tejedor, 2016; Shih \& Tsai, 2017). A partir de una triangulación de datos de los resultados mostrados, se concluye de un modo estructurado los mismos: 
- No se aprecian mejoras estadísticamente significativas respecto al rendimiento académico de estos enfoques (dimensión 1)

- El método de ABP y las TIC ayudan al aprendizaje del estudiante propiciando una mejora significativa (figura 1)

- Se destacan mejoras estadísticamente significativas en relación a la satisfacción del alumnado (dimensión 2)

- Se destacan mejoras estadísticamente significativas en relación a la motivación del alumnado (dimensión 3)

- Los alumnos valoran positivamente el uso e integración del ABP y las TIC (figura 2 y figura 3)

Aunque los alumnos que han utilizado el ABP y las TIC han mejorado los resultados respecto al grupo de control, las diferencias no son significativas, por lo que no se puede asegurar que el uso de esta metodología y recursos tecnológicos superen completamente los enfoques tradicionales utilizados en el grupo de control. En cambio, en las otras dos dimensiones de satisfacción y motivación si se detecta una mejora claramente significativa. Particularmente se destaca la participación activa centrada en el alumnado al trabajar grupalmente los contenidos, realizando experimentos, canciones y cuestionarios a expertos siendo el mismo muy favorable en el grupo experimental.

Cabe resaltar que se obtienen mejoras significativas en el reconocimiento de los contenidos de la higiene de las manos al manipulando objetos, realizando actividades a través de las TIC, realizando actividades colaborativas... Por ejemplo, debido al experimento, los alumnos entienden que las bacterias residen en cualquier lugar y la necesidad de una buena limpieza de manos. También, mediante el trabajo en grupo, los alumnos se ayudan unos a otros en caso de dudas, intercambiando información y debatiendo. Por ende, los alumnos investigan de primera mano reflexionando y realizando un aprendizaje significativo.

En este sentido, con los datos que se obtienen se destaca que los materiales son útiles en los procesos de enseñanza aprendizaje. Esta metodología, además, fomenta unas sesiones de trabajo divertidas, motivadoras y satisfactorias para el alumnado, propiciando el trabajo grupal, el análisis y la exploración de la higiene de las manos, de los contenidos del área de Ciencias Sociales.

En conclusión, no se aprecian mejoras significativas en el rendimiento académico al aplicar el ABP y las TIC. Sin embargo, se destacan otras ventajas muy importantes en relación a la motivación, satisfacción, diversión, curiosidad al hacer el experimento y utilidad de los materiales empleados desde una perspectiva didáctica. La ventaja de un experimento y de los videos e interacciones con los alumnos a través de las TIC, fomenta la comprensión de la importancia de la higiene de las manos.

En consecuencia, es el momento de promover el ABP y las TIC en el contexto de Educación Primaria, ya que la integración de metodologías centradas en el estudiante unidas a recursos llamativos y singulares para los estudiantes, abarcan una oportunidad única para la transformación educativa del siglo XXI. Como indican Dussel y Quevedo (2011), en la era digital el conocimiento en el aula está implícitamente conectado por el uso de las herramientas digitales tales como, móviles, tablets, ordenadores... Asimismo, la importancia de la correcta utilización de las metodologías de aprendizaje da sentido y justificación al manejo de las herramientas digitales (Prendes, Gutiérrez y Martínez 2018). 


\section{Referencias}

Area, M. (2015). De la enseñanza con libros de texto al aprendizaje en espacios online gamificados. Educatio Siglo XXI, 3, 15-38.

Ausubel, D.P., Novak, J.D., y Hanesian, H. (1983). Psicología Educativa. Un punto de vista cognitivo. México: Trillas.

Barroso, J., Cabero, J., Castaño, C., Llorente, M.d.C., Robero, R., y Román, P. (2007) Diseño y producción de TIC para la formación. Barcelona: UOC.

Blanchard, M., Glower, A. M., Jiménez, M., y Muzás, M. D. (2014). Transformando la sociedad desde las aulas. Metodología de aprendizaje por proyectos para la innovación educativa en El Salvador. Madrid: Narcea.

Brugia, M., \& Zukersteinova, A. (2019). Continuing vocational training in EU enterprises. Luxemburgo: Publications Office of the European Union. https://doi.org/10.2801/704583

Cohen, J. (1960). A coefficient of agreement for nominal scales. Educational and Pscychological Measurement, $20(1), 37-46$.

Cohen, S. (1998). Psychosocial models of the role of social support in the etiology of physical disease. Health Psychology, 7(3), 269-297.

Creswell, J.W. (2003). Research design: Qualitative, quantitative, and mixed methods approach. Thousand Oaks, CA: Sage.

Domínguez, C., \& Jaime, A. (2010). Database design learning: A project-based approach organized through a course management system. Computers \& Education, 55(3), 1312-1320. https://doi.org/10.1016/ j.compedu.2010.06.001

Domínguez, F.J. (2016). Redes sociales digitales y juventud universitaria: Un tema emergente en investigación educativa. Ensayos Pedagógicos, 0, 55-71.

Dussel, I., y Quevedo, L.A. (2011). Aprender y enseñar en la cultura digital. Buenos Aires: Fundación Santillana del Mar.

European Commision. (2016). DigCompOrg. Digitally Competent Educational Organisations.

European Parliament and council. (2006). Key Competences for Lifelong Learning-A European Framework. Official Journal of the European Union on 30 December 2006/L394.

Fernandes, S.R.G. (2014). Preparing graduates for professional practice: Findings from a case study of projectbased learning (PBL). Procedia-Social and Behavioral Sciences, 139, 219-226. https://doi.org/ 10.1016/j.sbspro.2014.08.064

Hair, J.F., Anderson, R.E., Tatham, R.L., \& Black, W.C. (1998). Multivariate data analysis (5th ed.). Upper Saddle River, New Jersey: Prentice Hall.

Helle, L., Tynjälä, P., \& Olkinuora, E. (2006). Project-based learning in post-secondary education theory, practice and rubber sling shots. Higher Education, 51(2), 287-314. https://doi.org/10.1007/ s10734-004-6386-5

Heo, H., Lim, K.Y., \& Kim, Y. (2010). Exploratory study on the patterns of online interaction and knowledge co construction in project-based learning. Computers \& Education, 55(3), 1383-1392. https://doi.org/ 10.1016/j.compedu.2010.06.012

INTEF (2017). Organizaciones Educativas Digitalmente Competentes.

Johnson, D.W. (2003). Social interdependence: interrelationships among theory, research, and practice. American Psychologist, 58(11), 934-945.

Johnson, G. (2006). Synchronous and asynchronous text-based CMC in educational contexts: a review of recent research. TechTrends: Linking Research and Practice to Improve Learning, 50(4), 46-53. 
Johnson, L., Adams Becker, S., Estrada, V., \& Freeman, A. (2015). NMCHorizon Report: 2015 K-12 Edition. Austin, Texas: The New Media Consortium.

Ley Orgánica 2/2006, de 3 de mayo, de Educación. (2006). BOE núm. 106, de 4 mayo 2006. Referencia: BOEA-2006-7899.

Ley Orgánica 8/2013, de 9 de diciembre, para la mejora de la calidad educativa. (2013). BOE núm. 295, de 10 de diciembre de 2013.

Michaelsen, L.K., Knight, A.B., \& Fink, D.L. (2004). Team-based learning: A transformative use of small groups. Westport, CT: Praeger.

Ministerio de Educación y Cultura y Deporte, MECD (2014). Real Decreto 126/2014, de 28 de febrero, por el que se establece el currículo básico de la Educación Primaria.

OCDE. (2005). La definición y selección de competencias clave.

Pérez, J.M., y Tejedor, S. (2016). Ideas para aprender a aprender. Manual de innovación educativa y tecnológica. Barcelona: UAB

Powell, P., \& Weenk, W. (2003). Project-led engineering education. Utrecht: Lemma Publishers.

Prendes, M.P., Gutiérrez, I., y Martínez, F. (2018). Competencia digital: una necesidad del profesorado universitario en el siglo XXI. RED: Revista de Educación a Distancia, (56), 1-22. http://dx.doi.org/ $10.6018 / \mathrm{red} / 56 / 7$

Sáez-López, J.M., y Cózar, R. (2017). Programación visual por bloques en Educación Primaria: Aprendiendo y creando contenidos en Ciencias Sociales. Revista Complutense de Educación, 28(2), 409-426. http:// dx.doi.org/10.5209/rev_RCED.2017.v28.n2.49381

Salinas, J. (2008). Innovación educativa y uso de las TIC. Sevilla: UNIA

Sendag, S., \& Odabasi, H.F. (2009). Effects of an online problem based learning course on content knowledge acquisition and critical thinking skills. Computers \& Education, 53(1), 132-141. https://doi.org/ 10.1016/j.compedu.2009.01.008

Shih, W.L., \& Tsai, C.Y. (2017). Students 'perception of a flipped classroom approach to facilitating online project-based learning in marketing research courses. Australasian Journal of Educational Technology, $33(5), 32-49$.

Thomas, J.W. (2000). A review of research on project-based learning. Novato, CA: The Buck Institute for Education.

UNESCO. (2011). UNESCO ICT Competency Framework for Teachers.

Williamson, B., Potter, J., \& Eynon, R. (2019). New research problems and agendas in learning, media and technology. Learning, Media and Technology, 44(2), 87-91. https://doi.org/ 10.1080/17439884.2019.1614953

Yang, S., \& Kwok, D. (2017). A study of students' attitudes towards using ICT in a social constructivist environment. Australasian Journal of Educational Technology, 33(5), 50-62. https://doi.org/10.14742/ ajet. 2890 